\title{
Macrosegregation Formation in an Al-Si Casting Sample with Cross-sectional Change During Directional Solidification
}

\author{
Haijie Zhang ${ }^{1}$ - Menghuai $\mathrm{Wu}^{1}$ • Yongjian Zheng ${ }^{1}$ Andreas Ludwig ${ }^{1}$ • \\ Abdellah Kharicha ${ }^{1}$
}

Received: 11 July 2018/Accepted: 6 September 2018/Published online: 6 October 2018

(C) The Author(s) 2018

\begin{abstract}
A unidirectional solidification experiment of hypoeutectic Al-7.0 wt\% Si alloy against gravity direction in a cylindrical mold with cross-sectional change was made, and the macrosegregation in different parts of the assolidified sample was investigated (Ghods et al. in J Cryst Growth 441:107-116, 2016; J Cryst Growth 449:134-147, 2016). The current study is to use a two-phase columnar solidification model to analyze the segregation mechanisms as used in this experiment. Following flow phenomena and their contributions to the formation of macrosegregation are simulated and compared: (1) solidification shrinkageinduced feeding flow; (2) thermo-solutal convection; and (3) combined thermo-solutal convection and shrinkage-induced feeding flow. The shrinkage-induced feeding flow leads to an inverse (positive) segregation in the bottom part, and a severe negative segregation in the part below cross-sectional change. Thermo-solutal buoyancy leads to a so-called steepling convection in the main part of the sample (away from the bottom and cross-sectional change), and this kind of flow leads to a positive macrosegregation near the sample surface. The calculations have successfully explained the experimental result of macrosegregation.
\end{abstract}

Keywords Macrosegregation · Fluid flow - Shrinkage · Directional solidification

Menghuai Wu

menghuai.wu@unileoben.ac.at

1 Chair for Simulation and Modeling of Metallurgy Processes, Department of Metallurgy, University of Leoben, 8700 Leoben, Austria

\section{Introduction}

Macrosegregation is caused by different flow phenomena during solidification [1-3]. To improve understanding of the macrosegregation mechanisms, unidirectional solidification experiments, based on $\mathrm{Al}-\mathrm{Si}$ and $\mathrm{Al}-\mathrm{Cu}$ alloys, were recently performed by Ghods [4-6]. One special feature of these experiments is that the cylindrical casting sample is designed with a cross-sectional change. Therefore, following flows and their impact on the macrosegregation formation can be quantitatively investigated: the solidification shrinkage-induced feeding flow, the thermo-solutal convection, and the flow pattern as caused by the sectionchange. The as-solidified structure is dominantly columnar. The current paper is to use a two-phase solidification model to "reproduce" the above experiments, with a focus on the Al-7.0 wt $\%$ Si alloy.

\section{Simulation Settings}

A volume-average-based solidification model has been introduced previously [7-9]. Here only two phases are considered: liquid and columnar. Fluid flow in the mushy zone is approximated with the Darcy's law, with its permeability being treated as a function of the primary dendrite arm space, $\lambda_{1}=500 \mu \mathrm{m}$ [4]. The columnar phase develops from the bottom of the sample, growing in the direction of temperature gradient. The feeding flow is due to the density difference between the liquid $\left(\rho_{\ell}=2408 \mathrm{~kg} / \mathrm{m}^{3}\right)$ and solid $\left(\rho_{s}=2545 \mathrm{~kg} / \mathrm{m}^{3}\right)$. The Boussinesq approximation has been employed to calculate the thermo-solutal convection, with the solutal $\left(\beta_{\mathrm{c}}\right)$ and thermal $\left(\beta_{\mathrm{T}}\right)$ expansion coefficients, $1.31 \times 10^{-3} \mathrm{wt}^{-1}$ and $-1.8 \times 10^{-4} \mathrm{~K}^{-1}$, respectively. Temperature fields in the casting sample and in the mold 
(graphite) are calculated, and an ideal mold-alloy contact is assumed for the heat transfer boundary condition.

As shown in Fig. 1, a graphite mold with section change is considered. The casting domain is filled with liquid melt of initial composition ( $\mathrm{Al}-7.0 \mathrm{wt} \% \mathrm{Si}$ ). Both the casting and mold are initialized with a uniform temperature (1490 K). The sample solidifies directionally with a fixed growth speed $(29.1 \mu \mathrm{m} / \mathrm{s})$ and temperature gradient $(G=5100 \mathrm{~K} / \mathrm{m})$. Other simulation settings are consistent with Ghods' experiments [4, 6]. Material properties refer to Ref. [4]. Two cases have been simulated: Case 1-solidification with the shrinkage-induced feeding flow only; and Case 2-solidification with combination of thermo-solutal convection and shrinkage-induced feeding flow. All simulations are performed in 2D axisymmetric, and a very fine mesh $(\Delta x=0.2 \mathrm{~mm})$ and a small time step $(\Delta t=0.002 \mathrm{~s})$ have been used.

\section{Results and Discussions}

\subsection{Case 1: Macrosegregation Caused by Shrinkage-Induced Feeding Flow Only}

Figure 2 shows the evolution of macrosegregation in the bottom region of sample (far away from the region of section change). As an ideal unidirectional solidification casting condition is applied at this initial stage of solidification, a so-called reverse segregation develops in the casting surface region (bottom). This work is to "reproduce" the early analytical solution of Flemings' [10]. As the initially formed columnar structure at the sample

Fig. 1 Geometry of the simulation domain

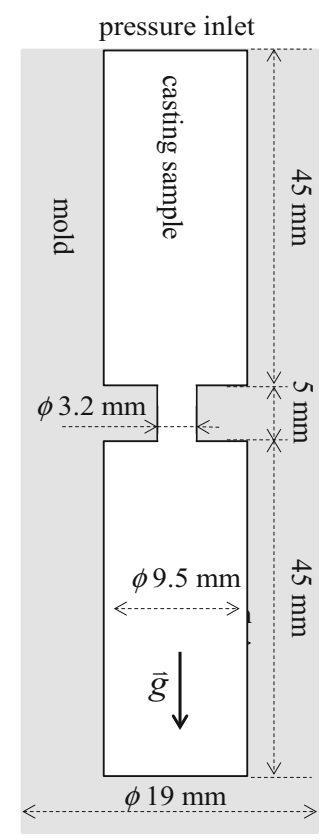

bottom is stationary, the solute-enriched melt in the mushy zone feeds the solidification shrinkage, leading to an increase in the mixture concentration $\left(c_{\text {mix }}\right)$ of the surface region. The maximum $c_{\text {mix }}$ reaches to 0.0703 , in comparison with the initial value of $0.07\left(c_{0}\right)$. The magnitude of feeding flow is small (Fig. 2b). In the region of uniform cross section (far away from the cross-sectional change), average velocity is $1.3 \mu \mathrm{m} / \mathrm{s}$, which reduces gradually to zero in the mushy zone. The maximum velocity (ca. $10 \mu \mathrm{m} / \mathrm{s}$ ) occurs in the region of cross section change of the sample. When the solidification proceeds to a steady state, e.g., at $900 \mathrm{~s}$, the change of $c_{\text {mix }}$ in the mushy zone (from initial value of $c_{0}$ to the minimum (0.06985) and then recovering to $c_{0}$ ), follows the typical evolution profile of $c_{\text {mix }}$ in the mushy zone of constant thickness [3, 10].

As the solidification front proceeds into the region of cross-sectional change (reduction) at $1800 \mathrm{~s}$, the magnitude of feeding flow reaches to $11 \mu \mathrm{m} / \mathrm{s}$, as shown in Fig. 3. A typical divergent flow pattern develops in the region below the cross-sectional reduction, leading to the formation of a strong negative $\left(c_{\text {mix }}<c_{0}\right)$ segregation in Zones I and II. The negative segregation extreme is about $c_{\text {mix }}=0.0675$ (Fig. 3b). Just below the shadow of the cross-sectional reduction (Zone III), a positive segregation forms, because the solidification shrinkage there needs to be fed by the solute-enriched melt.

\subsection{Case 2: Macrosegregation Caused by a Combination of Thermo-Solutal Convection and Shrinkage-Induced Feeding Flow}

As the thermo-solutal convection is considered, the magnitude of the liquid velocity is increased by 1-2 orders of magnitude, especially in the bulk region, i.e., the effect of flow on the macrosegregation formation is dominated by the thermo-solutal convection. At the initial stage $(t=366 \mathrm{~s})$ of solidification in the bottom region of sample, Fig. 4a, a clockwise vortex (right half of the sample) is developed just ahead of solidification front. Although the isotherms seem almost flat, the latent heat released by solidifying the first layer of solid from the bottom sample can only be extracted by the graphite mold, leading to the outward heat flux and inward flow pattern. The solute rejected from the columnar dendrite mushy zone is swept to the center of the sample, which enriches the $c_{\text {mix }}$ in the center part of the sample and depletes the $c_{\text {mix }}$ near the sample surface.

As solidification proceeds to a later stage $(t=1666 \mathrm{~s})$, Fig. $4 \mathrm{~b}$, the flow pattern changes. A quite portion of sample is solidified from the bottom sample. The thermal diffusivity $(185 \mathrm{~W} / \mathrm{mK})$ of the solid phase is roughly two times bigger than that $(76.7 \mathrm{~W} / \mathrm{mK})$ of the liquid. Now, the 


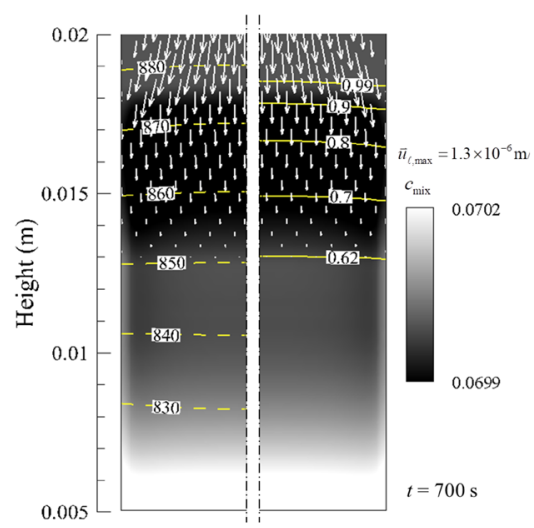

(a)

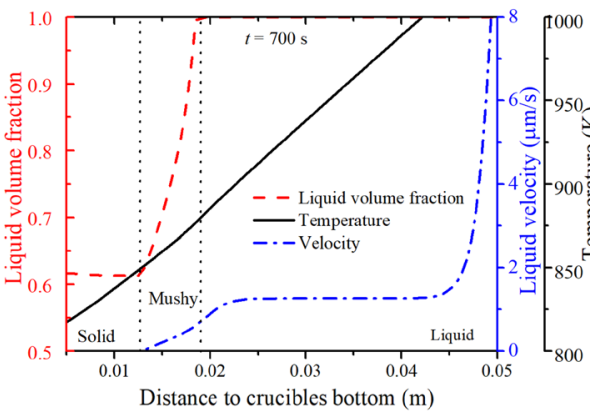

(b)

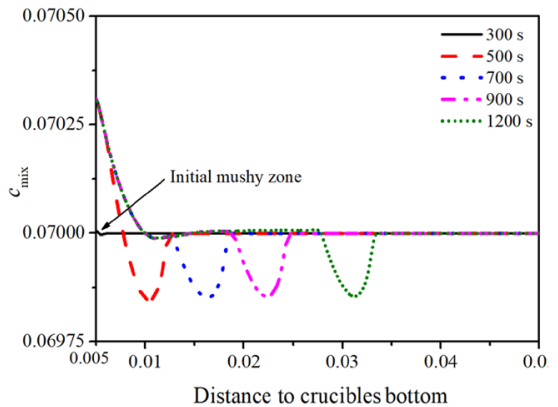

(c)

Fig. 2 Analysis of the inverse segregation in the bottom of the sample Case 1 . a The mixture concentration $\left(c_{\text {mix }}\right)$ in gray scale at $700 \mathrm{~s}$, overlaid with the liquid velocity in vectors, isotherms (left) and isolines of volume fraction liquid (right); $\mathbf{b}$ distribution profiles of volume fraction of liquid, temperature and liquid velocity along the axis at $700 \mathrm{~s}$; $\mathbf{c}$ evolution of macrosegregation $\left(c_{\text {mix }}\right)$ along the axis

Fig. 3 Analysis of macrosegregation formation in the region of cross-sectional change for Case 1. a The mixture concentration $\left(c_{\mathrm{mix}}\right)$ in gray scale at $1800 \mathrm{~s}$, overlaid with the liquid velocity in vectors and isotherms (the solidification front is indicated by a white solid line, $f_{\ell}=0.99$ ); b $c_{\text {mix }}$ profile along the axis at $t=2500 \mathrm{~s}$

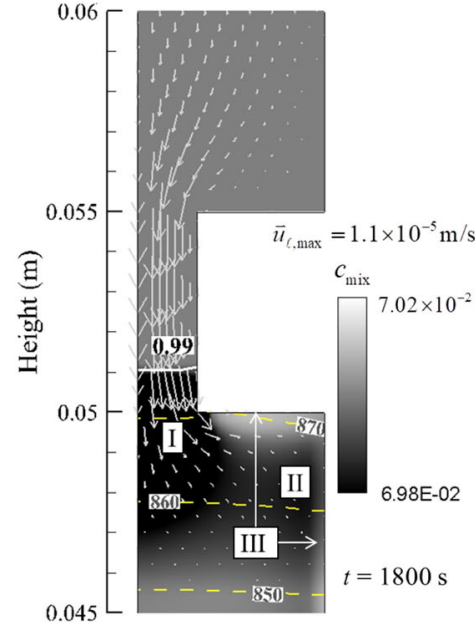

(a)

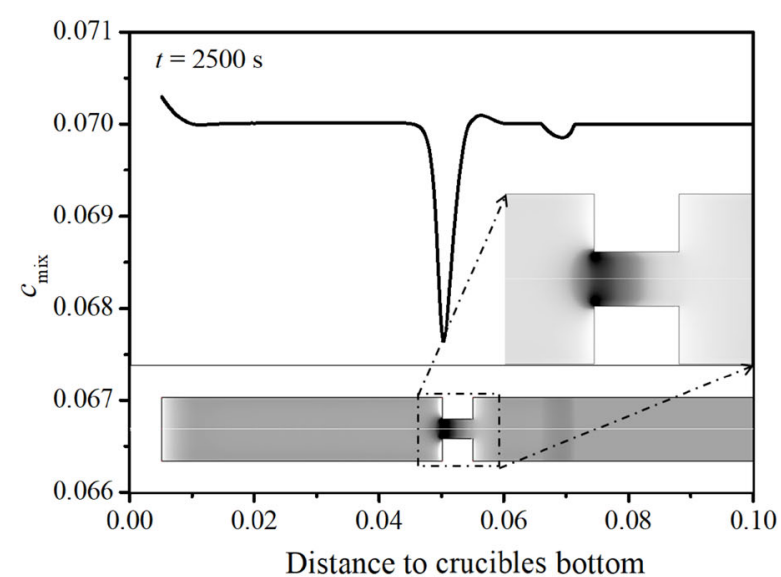

(b) isotherms are no longer flat, and the solidification front is bulged in the sample center. A so-called steepling convection is developed, i.e., the flow goes downward against the solidification front in the sample center and upward along the sample outer surface. Enriched solute in the mushy zone is swept to the periphery of the sample, leading to a positive segregation in the outer region of the casting surface and negative segregation in the casting center. The bulged solidification front in the sample center is the outcome of the macrosegregation: The denser soluteenriched melt concentrated in the periphery of the sample inhibits the solidification there.

Final segregation profiles along the radial and axial directions of the sample are shown in Fig. 5. The calculated segregation profile along the radial direction is found to agree almost perfectly with the experimentally measured result [4], except for one point at the casting surface. This is the only disagreement point at the sample surface which may be due to the experimental measurement method which has exaggerated the positive segregation there, or may be due to numerical model which has underestimated positive segregation by missing some other mechanisms.

It is worth mentioning that a further simulation (Case 3) is made, i.e., the macrosegregation is caused by the thermosolutal convection only (ignoring the shrinkage-induced feeding flow), which is compared with Case 2, as shown in Fig. 5b. The macrosegregation mechanism due to the thermo-solutal convection is dominant. The shrinkage-induced feeding flow enhances the negative segregation (or reduces the positive segregation) near the region of crosssectional change. 
Fig. 4 Analysis of macrosegregation formation for Case 2. a $c_{\text {mix }}$ distribution in gray scale at $t=366 \mathrm{~s}$, overlaid with liquid velocity in vectors and isotherms. b $c_{\text {mix }}$ distribution in gray scale at $t=1666 \mathrm{~s}$, overlaid with liquid velocity in vectors, isolines of $c_{\text {mix }}$ (left half) and isolines of liquid volume fraction (right half)

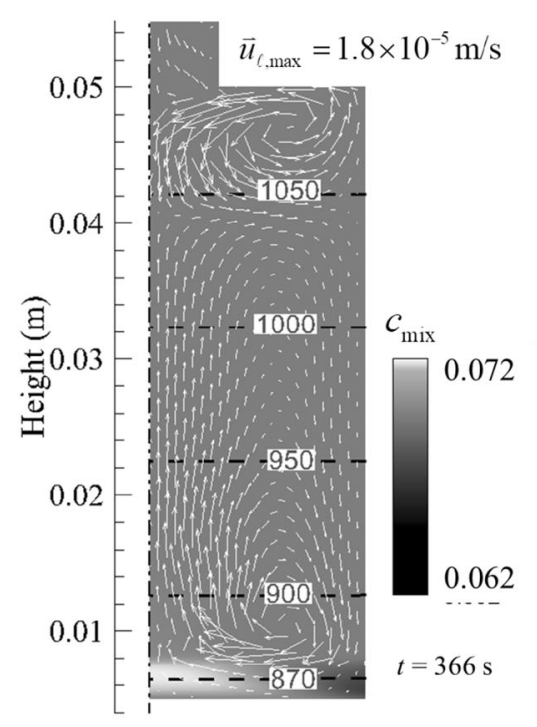

(a)

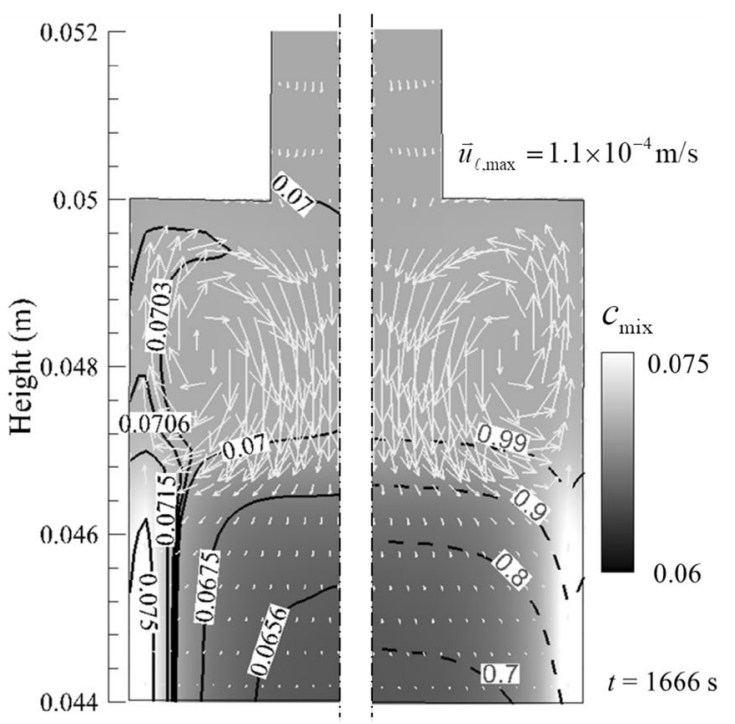

(b)

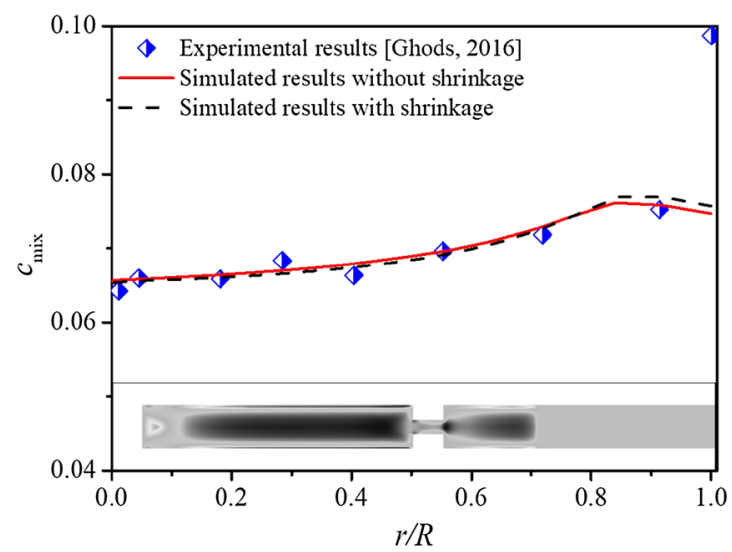

(a)

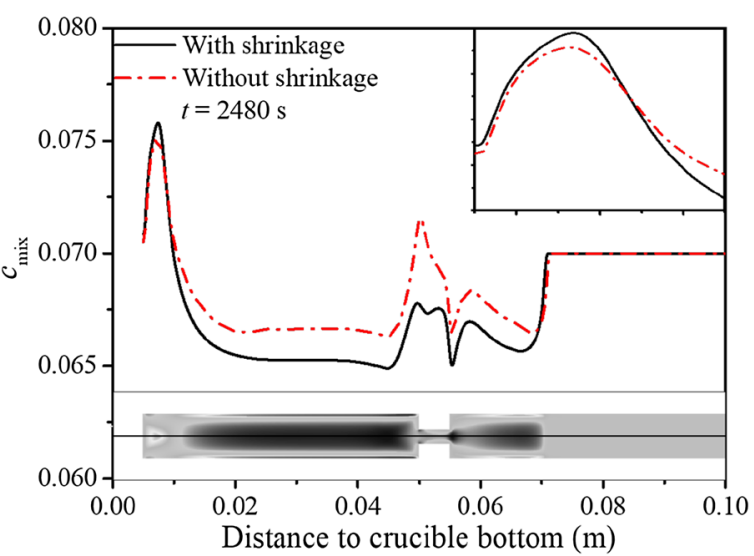

(b)

Fig. 5 Final macrosegregation $\left(c_{\text {mix }}\right)$ profiles along the radial (a) and axial (b) directions for Case 2. Comparison of the segregation profiles along the radial direction between simulation and experiment [4] is made

\section{Conclusions}

A two-phase solidification model has been used to "reproduce" the unidirectional solidification experiment with the Al-7.0 wt\%Si alloy and a casting sample of crosssectional change, as carried out by Ghods [4]. Numerical parameter studies have been made by considering different flow mechanisms. When only shrinkage-induced feeding flow is considered, the typical inverse macrosegregation in the sample bottom and a relatively strong negative segregation in the region below the cross-sectional change are predicted, which agrees with the classical work of Flemings [10]. Thermo-solutal buoyancy leads to a so-called steepling convection in the main part of the sample (away from the bottom and the region of cross-sectional change), and this kind of flow leads to a negative segregation in the sample center and positive macrosegregation in the periphery of the sample, which agrees with the experiment of Ghods [4].

\section{Acknowledgements}

Open access funding has been provided by Montanuniversität Leoben. The authors acknowledge the financial support from Austrian Research Promotion Agency (FFG)Austrian Space Application Program (ASAP) through the project FLOWSICONS (No. 859777), as well as the support from European Space Agency (ESA) through the project MICAST. 
Open Access This article is distributed under the terms of the Creative Commons Attribution 4.0 International License (http://creativecommons.org/licenses/by/4.0/), which permits unrestricted use, distribution, and reproduction in any medium, provided you give appropriate credit to the original author(s) and the source, provide a link to the Creative Commons license, and indicate if changes were made.

\section{References}

1. Flemings M C, Trans Iron Steel Inst Jpn 40 (2000) 838.

2. Beckermann C, Metall Rev 47 (2002) 243.
3. Ludwig A, Wu M, and Kharicha A, Metall Mater Trans A 46 (2015) 4854.

4. Ghods M, Johnson L, and Lauer M, J Cryst Growth 441 (2016) 107.

5. Ghods M, Johnson L, and Lauer M, J Cryst Growth 449 (2016) 134.

6. Ghods M, Lauer M, and Grugel R N, J Mater Eng Perform 26 (2017) 1.

7. Li J, Wu M, and Kharicha A, Comput Mater Sci 55 (2012) 419.

8. Li J, Wu M, and Kharicha A, Comput Mater Sci 55 (2012) 407.

9. Wu M, Zheng Y, A, and Kharicha, Comput Mater Sci 124 (2016) 444.

10. Flemings M C, and Nereo G E, Trans Metall Soc AIME 242 (1968) 41. 